Informe especial

\title{
Supervivencia de Vibrio cholerae 01 en agua dulce superficial y cólera endémico: una hipótesis geoecológica
}

\author{
René J. Borroto ${ }^{1}$
}

RESUMEN El peligro de que el cólera esté adquiriendo carácter endémico en países de América Latina hace necesario conocer la localización geográfica de los ambientes acuáticos que presentan condiciones ecológicas apropiadas para albergar Vibrio cholerae $O 1$ toxígeno biotipo El Tor. Para ello deberán realizarse muestreos de las aguas a fin de determinar en cuáles se encuentra este agente patógeno. Para hacer muestreos eficientes y eficaces sería útil saber qué cuerpos de agua presentan condiciones ecológicas favorables para la supervivencia del microbio durante períodos interepidémicos y cuál es su ubicación geográfica. En el presente trabajo se plantea la hipótesis de que las posibilidades de supervivencia de Vibrio cholerae O1 biotipo El Tor en cuerpos de agua dulce superficial tienden a ser inversamente proporcionales a la altura sobre el nivel del mar en que se localizan esos ambientes acuáticos.

La séptima pandemia de cólera afecta a América Latina desde enero de 1991 y se teme que adquiera carácter endémico. Según la OPS (1), en Perú, Ecuador y algunos países de Centroamérica ha aparecido una variante estacional de esta enfermedad que apunta a su posible carácter endémico en estos lugares.

Colwell et al. $(2,3)$ han postulado que el carácter endémico y estacional del cólera depende de la supervivencia de Vibrio cholerae $\mathrm{O} 1$ toxígeno en estado viable, pero no necesariamente cultivable, en nichos ecológicos localizados en reservorios acuáticos, durante pe-

\footnotetext{
1 Ministerio de Ciencia, Tecnología y Medio Ambiente, Instituto de Geografía Tropical, Departamento de Medio Ambiente, La Habana, Cuba. Dirección postal: Calle 11 número 514 entre D y E, Vedado, CP 10400, La Habana, Cuba.
}

ríodos interepidémicos. Según Glass y Black (4), para lograr un control eficaz del cólera es necesario evitar la exposición humana a esos reservorios. Ello implica localizar los ambientes acuáticos cuyas características ecológicas favorecen la proliferación de este microorganismo patógeno durante largos períodos y tomar muestras de las aguas.

\section{El aislamiento de Vibrio cholerae}

El aislamiento de $V$. cholerae mediante métodos de cultivo a partir de muestras tomadas en ambientes acuáticos, y su detección mediante microscopia directa, métodos inmunológicos o técnicas de biología molecular, son caros y de aplicación limitada en países en desarrollo. Para poder reali- zar muestreos eficientes y adecuados, sería útil determinar primero qué cuerpos de agua presentan las condiciones ecológicas más favorables para la supervivencia del microorganismo durante períodos interepidémicos.

Debido a que el agua dulce superficial se bebe con frecuencia, especialmente entre quienes carecen de acceso a servicios de agua potable, se cree (3, 5-7) que puede desempeñar una función importante como vehículo transmisor del agente causal en la aparición de casos primarios de cólera o de sus portadores asintomáticos. ${ }^{2}$ Los mejores puntos de muestreo para aislar o detectar este microorganismo en

\footnotetext{
2 Hasta el presente, no se ha planteado que el Vibrio cholerae O1 toxígeno biotipo El Tor tenga nichos ecológicos en aguas subterráneas, donde logre sobrevivir durante períodos interepidémicos.
} 
ambientes acuáticos suelen ubicarse en zonas de gran contaminación fecal humana. Sin embargo, $V$. cholerae O1 toxígeno biotipo $\mathrm{El}$ Tor puede sobrevivir durante períodos interepidémicos en ausencia de contaminación fecal humana, tanto en aguas de estuarios como en aguas dulces $(5,6)$. Por tal motivo también es necesario tomar muestras en lugares sin contaminación fecal humana, bien sean aguas saladas, medianamente salinas, o dulces.

\section{Condiciones que favorecen la proliferación de Vibrio cholerae a mayores alturas}

Para ubicar los puntos de muestreo es fundamental preguntarse ¿qué cuerpos de agua dulce superficial reúnen las condiciones ecológicas más favorables para la supervivencia del vibrión durante períodos interepidémicos, incluso en ausencia de contaminación fecal humana? En el presente trabajo se plantea la hipótesis de que las posibilidades de $V$. cholerae O1 toxígeno biotipo El Tor de sobrevivir en cuerpos de agua dulce superficial tienden a relacionarse inversamente con la altura sobre el nivel del mar de esos ambientes acuáticos. A mayor altura, el microorganismo enfrenta condiciones más adversas, entre las cuales figuran las siguientes:

- Temperaturas bajas: La temperatura óptima para que crezca el vibrión oscila entre 30 y $37{ }^{\circ} \mathrm{C}$; el crecimiento se inhibe a temperaturas menores de $15^{\circ} \mathrm{C}$. Debido a la caída de la temperatura a medida que aumenta la altura, el agua de ríos, lagos y embalses es más fría en zonas montañosas que en zonas llanas cerca del nivel del mar.

- Un menor contenido de materia orgánica y nutrientes: $V$. cholerae $\mathrm{O} 1$ toxígeno requiere carbohidratos, nitrógeno, azufre, fósforo y sodio para su metabolismo. El agua que fluye en el tercio superior de los ríos tiende a ser más limpia y a tener menos materia orgánica y nutrientes que el agua que fluye en el tercio inferior porque las corrientes suelen ser más veloces en zonas montañosas. Esto se debe a que la inclinación promedio de la pendiente es mayor que en zonas llanas. A mayor velocidad, la oxigenación y autodepuración natural del agua pueden ser más eficaces $(8,9)$. Los nutrientes y la materia orgánica que no logran depurarse aguas arriba se suman a los vertidos que ingresan en los ríos aguas abajo. De ahí que sus concentraciones tiendan a ser mayores en el tercio inferior del río que en el tercio superior. Por otra parte, en ecosistemas de aguas lentas (estancadas), tales como lagos y embalses, la concentración de nutrientes también tiende a ser menor hacia las cotas más altas del relieve y viceversa. Aunque la eutrofización ${ }^{3}$ depende en gran medida de los vertidos de residuales y del uso de fertilizantes inorgánicos por el hombre, la de origen natural tiende a ser menor en los lagos y embalses de zonas montañosas que en los situados en llanuras bajas.

- Poca salinidad: El nivel óptimo de salinidad para el crecimiento del vibrión del cólera fluctúa entre 15 y 25 partes en 1 000. Estas concentraciones suelen encontrarse en los ambientes acuáticos de zonas bajas, como estuarios, ciénagas y pantanos costeros. En el agua dulce, cuyo nivel de salinidad es menor de 1 en 1000 partes, $V$. cholerae solo puede crecer si existen suficientes nutrientes orgánicos o cationes divalentes de calcio o magnesio que permitan compensar la poca disponibilidad de ion sodio. Como estos nutrientes son transportados por las corrientes aguas abajo, sus concentraciones tienden a ser menores a mayor altura.

- Mayor intensidad de la radiación ultravioleta: Dado que el microorganismo es muy susceptible a esta radiación, se ha propuesto (10) estudiar el uso de la luz solar para desinfectar el agua de beber en la cordillera de Los Andes. El efecto

\footnotetext{
3 La eutrofización es el enriquecimiento de un cuerpo de agua con nutrientes, especialmente nitratos y fosfatos.
}

atenuante que ofrece la atmósfera al paso de la radiación ultravioleta tiende a ser menor en zonas montañosas que en zonas llanas cercanas al nivel del mar, debido a que las montañas tienen menos turbidez atmosférica producida por aerosoles y una menor concentración de oxígeno.

- Corrientes más turbulentas: Las corrientes de aguas superficiales tienden a ser más rápidas y turbulentas en zonas montañosas que en zonas llanas. La captación de nutrientes por parte del vibrión del cólera se dificulta en ambientes turbulentos y se facilita en aguas tranquilas.

Una causa de estrés importante es el $\mathrm{pH}$, dado que el vibrión del cólera es muy sensible a la acidez. El pH en ambientes acuáticos no muestra, sin embargo, una clara asociación con la altura. Puede ser alcalino, neutro o ácido, tanto en zonas bajas como altas, y depende en gran medida de las concentraciones de carbonatos, bicarbonatos y dióxido de carbono.

\section{La obtención de nutrientes por Vibrio cholerae y la altura}

En respuesta a condiciones de estrés ambiental, el agente patógeno adopta un estado que los microbiólogos ambientales han definido como viable no cultivable (VNC) (4): viable porque mantiene la capacidad de realizar funciones metabólicas y formar colonias; no cultivable porque no puede aislarse mediante métodos de cultivo en el laboratorio. Si las condiciones ambientales vuelven a ser favorables, el microorganismo puede retornar al estado cultivable. En dicho estado o en estado VNC se adhiere a la superficie de especies de fitoplancton (particularmente las algas verdes y verdiazules) y zooplancton (especialmente los copépodos), concentrándose en ellas, así como al intestino de peces y a la superficie quitinosa de ostras, cangrejos y camarones (6). También se adhiere a las raíces de plantas macrófitas de agua dulce, tales como Eichhornia crassipes y Lemna minor, y a la superficie de 
sedimentos de materias orgánicas e inorgánicas en los cuales el vibrión puede encontrar nutrientes para su metabolismo.

A mayor altura, el plancton tiende a crecer menos debido a los siguientes factores: a) En cuerpos de agua dulce superficial situados en zonas montañosas, el contenido de nutrientes esenciales para el crecimiento del fitoplancton, particularmente los nitratos y fosfatos, tiende a ser menor que en zonas bajas receptoras del drenaje procedente de cotas superiores; b) los días despejados (sin nubes y soleados) tienden a ser menos frecuentes en zonas montañosas que en lugares llanos. El sol tarda más en aparecer y menos en ponerse en un relieve montañoso que en uno llano donde no existen montañas que obstaculicen los rayos solares. Como resultado, el número de horas de luz en zonas montañosas es menor, lo cual limita el proceso de fotosíntesis necesario para que prolifere el fitoplancton. Por el contrario, la intensa actividad biológica de la radiación ultravioleta - cuyo nivel suele ser superior a mayor altura- limita el crecimiento del plancton; c) las bajas temperaturas de las aguas en zonas montañosas inhiben el crecimiento del fitoplancton, especialmente de algas verdes y verdiazules, que requieren temperaturas entre 25 y $35{ }^{\circ} \mathrm{C}(11)$; d) el plancton suele crecer menos en aguas de flujo rápido propias de las zonas montañosas y más en aguas estancadas o de flujo lento, y cuando logra multiplicarse en corrientes rápidas, es arrastrado aguas abajo.

La macrofauna acuática (peces, moluscos, crustáceos) también suele ser más abundante y diversa en aguas dulces superficiales cercanas a las cotas más bajas del relieve (8) porque en ellas hay más plancton, componente esencial de la cadena alimentaria de la macrofauna acuática. Las macrófitas de agua dulce, como E. crassipes y $L$. minor - a ellas se adhiere $V$. cholerae O1 toxígeno- son plantas flotadoras que habitan en aguas estancadas o de muy lento movimiento. Necesitan nutrientes, especialmente nitratos $\mathrm{y}$ fosfatos (8), que obtienen con más facilidad en cuerpos de agua situados en llanuras bajas.

La posibilidad de una asociación del microorganismo con sedimentos de materias de origen orgánico e inorgánico como mecanismo para obtener nutrientes también es mayor en zonas llanas bajas, ya que las aguas de ríos y corrientes superficiales transportan partículas suspendidas (arcillas, limo, arenas y materia orgánica en suspensión) o materiales disueltos (iones fosfato, nitrato, calcio, nitrito, magnesio y materia orgánica en disolución) que se depositan y sedimentan aguas abajo. Por lo tanto, a mayor altura, las condiciones ecológicas que permiten que sobreviva $V$. cholerae $\mathrm{O} 1$ toxígeno biotipo $\mathrm{El}$ Tor en cuerpos de agua dulce superficial tienden a ser menos favorables, y a menor altura sucede lo contrario.

\section{COMENTARIO FINAL}

Nuestra hipótesis es deductiva y de carácter general y debe ponerse a prueba mediante investigaciones empíricas. Ya existe por lo menos un estudio, realizado por Tamplin y Carrillo (12), cuyos resultados la respaldan. Estos autores, en su búsqueda de reservorios acuáticos de $V$. cholerae, tomaron muestras de agua del lago Titicaca en los Andes, a más de 3000 metros sobre el nivel del mar; del río Amazonas en la zona del bosque tropical lluvioso; en la costa del océano Pacífico, y en el río Rimac, cerca de Lima, a pocos metros sobre el nivel del mar. $V$. cholerae O1 fue aislado con mayor frecuencia y en mayores concentraciones en las aguas tibias de los ríos Amazonas y Rimac que en las aguas frías $\left(12{ }^{\circ} \mathrm{C}\right)$ del lago Titicaca.

Para confirmar la hipótesis se necesitarán, sin embargo, más investigaciones que tomen en cuenta la magnitud y las variaciones estacionales de los parámetros abióticos (temperatura, nutrientes, salinidad) y bióticos (especies de fitoplancton, zooplancton, macrófitas acuáticas, peces, moluscos y crustáceos), relacionándolos con la presencia y las concentraciones del microorganismo en diferentes ambientes acuáticos (a diferentes alturas). Si la hipótesis quedara confirmada, sería útil realizar investigaciones epidemiológicas para conocer cuán protegidas contra la enfermedad o la infección asintomática están las poblaciones que utilizan aguas - para consumo, baño, lavado, y otros propósitos - donde el microbio no puede sobrevivir o se encuentra en concentraciones muy bajas, como resultado de la acción de factores de estrés que impiden que crezca o sobreviva. Las notables diferencias geoecológicas y climáticas observadas en América del Sur y Centroamérica favorecen la realización de esas investigaciones. Para cumplir tal propósito, los microbiólogos y epidemiólogos deberán unir sus esfuerzos a los de geógrafos y ecólogos del continente.

Agradecimiento. El autor agradece a Winny Suárez y Elibel Atala su revisión de este trabajo; a Anwarul Huq, Bohumil Drasar, Mark Tamplin, Mohammad Sirajul Islam, Paul Epstein, Roger Glass, David Sack y Richard Guerrant, el generoso donativo de trabajos actualizados de los cuales son autores; y a María Isabel González, su revisión y sugerencias.

\section{REFERENCIAS}

1. Organización Panamericana de la Salud. La situación del cólera en las Américas. Bol Epidemiol 1994;15(1):13-16.

2. Colwell R, Kaper J, Joseph S. Vibrio cholerae, Vibrio parahaemolyticus, and other vibrios: occurrence and distribution in Chesapeake Bay. Science 1977;198(4315):394-396.

3. Colwell R, Huq A. Vibrios in the environment: Viable but non-culturable Vibrio cholerae. En: Kaye Wachsmuth I, Blake P, Olsvik $\varnothing$, eds.
Vibrio cholerae and cholera: Molecular to global perspectives. Washington, DC: American Society for Microbiology; 1994. pp. 117-133.

4. Glass R, Black R. The epidemiology of cholera. En: Barua D y Greenough III W, eds. 
Cholera. New York: Plenum Medical Company; 1992. pp. 129-154.

5. Islam MS, Drasar B, Bradley Sack R. Probable role of blue-green algae in maintaining endemicity and seasonality of cholera in Bangladesh: A hypothesis. J Diarrh Dis Res 1994; 12(2):245-256.

6. Islam MS, Drasar B, Bradley Sack R. The aquatic flora and fauna as reservoirs of Vibrio cholerae: A review. J Diarrh Dis Res 1994;12(2): 87-96.

7. Huq A, Xu B, Chowdhury M, Islam MS, Montilla $R$, Colwell R. A simple filtration method to remove plankton-associated Vibrio cholerae in raw water supplies in developing countries. Appl Environ Microbiol 1996;62(7): 25082512.

8. Nebel B, Wright R. Environmental science: The way the world works. 4th edition. New Jersey: Prentice Hall; 1993.

9. Tyler Miller Jr G. Environmental science: Sustaining the earth. 3rd edition. California: Wadsworth Publishing Company; 1991.

10. Tauxe R, Blake P. Epidemic cholera in Latin America. J Am Med Assoc 1992;267(10):13881390.
11. ReVelle P, ReVelle C. The environment: Issues and choices for society. 1st edition. New York: D. Van Nostrand Company; 1981.

12. Tamplin M, Carrillo C. Environmental spread of Vibrio cholerae in Perú [carta al editor]. Lancet 1991;338(8776):1216-1217.

Manuscrito recibido el 30 de abril de 1997 y aceptado para publicación en versión revisada el 13 de abril de 1998.
ABSTRACT

The survival of Vibrio cholerae

01 in surface freshwater and endemic cholera: A geoecological hypothesis
The danger that cholera is becoming endemic in Latin America makes it imperative to know the geographic location of aquatic environments where ecological conditions favor long-term survival of the toxigenic Vibrio cholerae O1 El Tor biotype, and such aquatic environments should be sampled to determine if they harbor this microorganism. For efficient and effective sampling, it would be useful to know what kinds of waters are ecologically suitable for the survival of this pathogen during periods between epidemics, and where these bodies of water are located. This paper presents the hypothesis that toxigenic $V$. cholerae O1's ability to survive in surface freshwaters tends to be inversely related to the altitude above sea level of these freshwaters. 